Immer mehr Menschen mit Diabetes auf Station

\title{
Wann gibt es leitliniengestützte Glukosezielwerte für die Klinik?
}

Liebe Leserinnen und Leser,

wie die neuesten Zahlen der Internationalen Diabetes Federation (IDF) von Ende des Jahres 2009 gezeigt haben, ist die Diabetesprävalenz weltweit weiter deutlich angestiegen. Deutschland nimmt mit 12\% Diabetesprävalenz in der Gesamtbevölkerung in Europa einen traurigen ersten Platz ein. Daraus lässt sich klar ableiten, dass eine frühzeitige und optimal strukturierte Behandlung von Menschen mit Diabetes sowohl kurz- als auch vor allem langfristig einen immensen Stellenwert hat. Es signalisiert darüber hinaus, dass eine erfolgreiche und auch bezahlbare Diabetestherapie nur im Rahmen einer von allen Beteiligten gelebten und optimal strukturierten, sektorenübergreifenden Versorgung geleistet werden kann.

\section{Blutzuckerwert bei stationärer Aufnahme beeinflusst Prognose}

Die hohe Diabetesprävalenz führt zwangsläufig dazu, dass immer mehr Menschen aufgrund verschiedener Erkrankungen oder wegen notwendiger Operationen in Kliniken behandelt werden, die zusätzlich einen Diabetes mellitus haben.

Bis vor Kurzem wurde eine normnahe Blutzuckereinstellung im Rahmen eines stationären Klinikaufenthaltes für nicht wichtig erachtet und die Hyperglykämie ausschließlich als Konsequenz der akuten Erkrankungssituation, eben als medizinischer Stress, beurteilt. Inzwischen belegt eine sehr umfangreiche Datenlage, dass die Höhe des Blutzuckers bei Aufnahme ins Krankenhaus entscheidenden Einfluss auf den Heilungsprozess, auf das „medizinische Outcome" nimmt und die Prognose signifikant verschlechtern kann (s. CME-Beitrag ab S. 34). Allerdings hat erst in der jüngeren Vergangenheit ein gewisses Umdenken eingesetzt und dazu geführt, dass ein strukturiertes Hyperglykämiemanagement in Kliniken eingeführt wurde. Idealerweise wird es durch ein interdisziplinäres Team unterstützt (Diabetologe, Diabetesberater, Ernährungsberater), beginnt bereits auf der Intensivstation und wird anschließend auf den Normalstationen fortgeführt.

\section{In der Klinik ist ein an Leitlinien orientiertes Diabetesmanagement nötig}

Patientinnen und Patienten, die von Anfang ihres Klinikaufenthaltes an ein optimales Hyperglykämiemanagement erfahren haben, haben eindeutig einen besseren medizinischen Verlauf und eine deutlich günstigere Prognose als jene, bei denen dies nicht der Fall ist. Zusätzlich können über eine auf diesem Weg verringerte Komplikationsrate auch die Gesamtkosten gesenkt werden. Daher ist es zwingend erforderlich, ein an Leitlinien ausgerichtetes strukturiertes Vorgehen mit guter Definition der Blutzuckerzielwerte für Patientinnen und Patienten in der Klinik zu entwickeln und flächendeckend zu etablieren.

Das innerhalb der Klinik umgesetzte interdisziplinäre Hyperglykämiemanagement, das konservative wie operative Kliniken sowie Versorgungseinheiten umfasst, könnte ein gutes Beispiel für die ebenfalls so dringend notwendige Etablierung einer besser strukturierten sektorenübergreifenden gemeinsamen Versorgung unserer Patientinnen und Patienten mit Diabetes mellitus sein.

Petra-Maria Schumm-Draeger

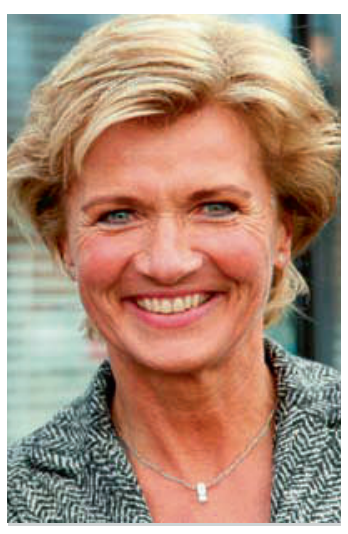

Prof. Dr. med. Petra-Maria Schumm-Draeger, Chefärztin am Klinikum Bogenhausen in München und Herausgeberin von Info Diabetologie 